\title{
Caracterização das águas subterrâneas usadas para irrigação na área produtora de melão da Chapada do Apodi ${ }^{1}$
}

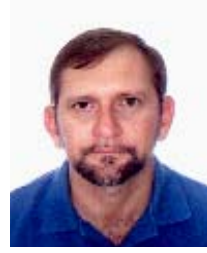

José F. de Medeiros ${ }^{2}$, Rodrigo de A. Lisboa ${ }^{3}$, Maurício de Oliveira ${ }^{4}$, Manoel J. da Silva Júnior ${ }^{3} \&$ Leonardo P. Alves ${ }^{3}$

\footnotetext{
1 Projeto financiado pelo Programa Avança Brasil em convênio com EMBRAPA/ESAM

2 Eng. Agr., D.Sc., Departamento de Engenharia Agrícola - ESAM, CP 137, CEP 59625-900, Mossoró, RN (Foto)

${ }^{3}$ Graduando em Agronomia; Bolsista do PIBIC/CNPq - ESAM

${ }^{4}$ Prof. Adj., D.Sc., Departamento de Solos e Geologia - ESAM
}

Protocolo 67 - 18/6/2000 - Aprovado em 24/9/2003

\begin{abstract}
Resumo: Objetivando-se estudar a qualidade da água de irrigação, em especial do aqüífero situado no calcário da formação Jandaíra, na região produtora de melão localizada na Chapada do Apodi, realizou-se este trabalho utilizando-se resultados de 45 análises completas $\left(\mathrm{CE}_{\mathrm{a}}, \mathrm{pH}\right.$ e concentrações de $\mathrm{Na}^{+}, \mathrm{Ca}^{2+}, \mathrm{Mg}^{2+}, \mathrm{K}^{+}, \mathrm{Cl}^{-}, \mathrm{HCO}_{3}{ }^{-}$e $\mathrm{CO}_{3}{ }^{2-}$ ) de amostras d'água de diversas localidades, inseridas nos municípios de Mossoró e Baraúna, e circunvizinhos, coletadas no período seco. As águas dos poços tubulares usadas para irrigação apresentaram níveis elevados de salinidade, $C_{a}$ variando de 1,25 a 3,00 dS m $\mathrm{m}^{-1}$. Existem diferentes regiões, cada uma com valores de salinidade, composição da água e riscos de obstrução específicos e diferenciados. Em algumas áreas, a salinidade e a toxicidade do cloreto podem afetar, de forma significativa, o rendimento de culturas sensíveis. As águas apresentam baixa sodicidade e elevada alcalinidade.
\end{abstract}

Palavras-chave: qualidade da água, alcalinidade, salinidade

\section{Characterization of the groundwater used for irrigation in the melon producing area of the Apodi Plateau}

\begin{abstract}
This research had the objective of studying the quality of the irrigation water, especially confined to Jandaira calcareous aquifer, in melon producing region of the municipal districts of Mossoró, Barauna and other surrounding areas. The results of 45 complete analyses (electrical conductivity, $\mathrm{pH}$ and concentrations of $\mathrm{Ca}^{2+}, \mathrm{K}^{+}, \mathrm{Cl}^{-}, \mathrm{HCO}_{3}^{-}$and $\mathrm{CO}_{3}{ }^{2-}$ ) of water samples belonging to different places, located in the study region were used. The water used for the irrigation collected from tubular wells presented high levels of salinity, varying from 1.25 to $3.00 \mathrm{dS} \mathrm{m}^{-1}$. Different areas exist, each one with different salinity values and obstruction risks. In some areas, the salinity and toxicity of the chloride may affect in a significant way the yield of sensitive crops. All analyzed water samples present low sodicity and high alkalinity.
\end{abstract}

Key words: water quality, salinited, alkalinity

\section{INTRODUÇÃO}

No Nordeste existem milhares de poços cujas águas são utilizadas para a irrigação, representando um importante insumo na cadeia produtiva; no entanto, o uso de água de má qualidade pode trazer danos ao meio ambiente, com sérios reflexos socioeconômicos (Silva Júnior et al., 1999).

Os municípios de Mossoró, Baraúnas e vizinhos a estes, no Estado do Ceará, que se localizam na Chapada do Apodi vêm, ultimamente, se destacando na produção de frutas e hortaliças irrigadas, sobretudo melão, produzindo tanto para o mercado interno como para o externo.
A principal fonte de água para a irrigação na Chapada do Apodi é subterrânea, cuja captação é feita do arenito Açu, em poços com cerca de $1000 \mathrm{~m}$ de profundidade, e do calcário Jandaíra, com profundidade em torno de $100 \mathrm{~m}$. Atualmente, o tipo de poço mais utilizado é aquele que explora o aqüífero calcário.

Segundo Medeiros (1992), Oliveira \& Maia (1998), entre outros, as águas do aqüífero calcário apresentam concentrações de sais relativamente elevadas, podendo ser superior a 2000 $\mathrm{mg} \mathrm{L}^{-1}\left(3,0 \mathrm{dS} \mathrm{m}^{-1}\right)$. Neste caso, sua utilização fica condicionada à tolerância das culturas à salinidade e ao manejo da irrigação, com vistas ao controle da salinização dessas áreas. 
A qualidade das águas dos poços utilizadas para a irrigação, pode variar no tempo e no espaço, e se sabe que na época de estiagem seus níveis de sais são mais elevados, quando também são mais altas as temperaturas e a evapotranspiração na região, com as culturas exigindo maior suprimento de água, a fim de atender às suas necessidades fisiológicas, sendo afetadas, portanto, com maior intensidade; assim, observa-se a importância do conhecimento de sua qualidade para evitar os problemas conseqüentes (Costa, 1982; Laraque, 1989; Medeiros, 1992; e Silva Júnior et al., 1999).

Segundo Oliveira \& Maia (1998) nos aqǘferos da região da Chapada do Apodi a água de poços tubulares rasos (influenciados pelo calcário) e profundos (confinados no arenito) apresenta maiores problemas que as águas superficiais, devido aos maiores valores de $\mathrm{pH}$ e à concentração de íons bicarbonatos.

A alcalinidade excessiva das águas destinadas à fertirrigação pode criar uma série de inconvenientes, que vão desde o entupimento dos emissores, pela precipitação de carbonatos e fosfatos, até a redução da disponibilidade de micronutrientes para as culturas (Egreja Filho et al., 1999).

Segundo Oliveira \& Maia (1998) as determinações de pH e $\mathrm{CE}_{\mathrm{a}}$ (condutividade elétrica) fornecem subsídios para se avaliar a possibilidade de precipitação de sais e a indução da salinidade em função da prática da irrigação; a relação de adsorção de sódio (RAS) assume papel preponderante, posto que a combinação CE e RAS serve para avaliar os perigos que a água oferece, respectivamente, em termos de indicação de salinidade e aumento dos teores de sódio na solução do solo e, consequentemente, problemas de infiltração; o uso da RAS, como proposto originalmente pelo Laboratório de Salinidade de Solo dos Estados Unidos (Richards, 1954) tem sido, no entanto, freqüentemente criticado, por subestimar o risco de sodicidade e por não levar em consideração outros íons $\left(\mathrm{HCO}_{3}^{-} \mathrm{e} \mathrm{CO}_{3}{ }^{2-}\right)$ sugerindo-se métodos de cálculos alternativos, como a RAS ajustada (RASaj) e da RAS corrigida (RAScor) (Ayers \& Westcot, 1999).

Sendo a maioria das culturas exploradas na região classificadas como sensíveis a moderadamente sensíveis à salinidade, surge a necessidade urgente de melhor caracterizar essas águas, para se estabelecer o manejo adequado das plantas, solo e sistemas de irrigação.

Com o presente trabalho, propõe-se estudar a qualidade da água de irrigação, sobretudo a de origem calcária, na região produtora de melão dos municípios de Baraúnas e Mossoró, e municípios vizinhos, no Estado do Ceará.

\section{MATERIAL E MÉTODOS}

O presente trabalho de campo foi realizado nos municípios de Mossoró, Baraúnas, RN, e em outros circunvizinhos, estes no Estado do Ceará, fazendo-se visitas nas empresas e propriedades rurais que trabalham com a produção de frutas e hortaliças irrigadas na região, e que possuem poços tubulares explorando o calcário Jandaíra. Em partes dos poços, representando todas as áreas visitadas, foram coletadas 45 amostras de água e encaminhadas aos Laboratórios de Irrigação e Salinidade e de Química e Fertilidade de Solos, respectivamente, dos Departamentos de Engenharia Agrícola e de Solos e Geologia da Escola Superior de Agricultura de Mossoró, ESAM, onde foram, de forma sistemática, caracterizadas quimicamente. Utilizando-se o mapa da região (SUDENE, 1972) fez a localização dos poços, determinando-se as suas coordenadas (latitude e longitude).

As amostras de água foram coletadas no período seco, durante os meses de setembro de 1999 e janeiro de 2000, observando-se os critérios para análise, conforme Palacios \& Aceves (1970) acondicionando-as em garrafas plásticas, opacas, de $500 \mathrm{~mL}$, hermeticamente fechadas e conduzidas para análise laboratorial. Nas amostras coletadas determinou-se $\mathrm{CE}_{\mathrm{a}}$, $\mathrm{pH}$ e concentrações de $\mathrm{Na}^{+}, \mathrm{Ca}^{2+}, \mathrm{K}^{+}, \mathrm{Cl}^{-}, \mathrm{HCO}_{3}^{-}$e $\mathrm{CO}_{3}{ }^{2-}$, de acordo com as metodologias propostas por Richards (1954) e EMBRAPA (1997). Realizou-se, também, o cálculo da RAS, RASaj e RAScor, segundo Medeiros \& Gheyi (1997) e Ayers \& Westcot (1999). Em todas as amostras de água foram determinados a $\mathrm{CE}_{\mathrm{a}}$ e o $\mathrm{pH}$ em campo, com o auxílio do condutivímetro e medidor de $\mathrm{pH}$ (momento da coleta). Os valores de $\mathrm{CE}$ e $\mathrm{pH}$ assumidos nas análises foram aqueles determinados em campo, primeiro porque os valores de CE foram similares e, secundo, pelo fato do $\mathrm{pH}$ medido no campo representar melhor as condições reais.

Depois de realizadas as análises foi feita comparação das características das águas entre os poços e localidades vizinhas, de modo a se verificar semelhança entre elas e estabelecer uma regionalização das águas. A partir deste agrupamento, fez-se uma estatística para cada parâmetro medido $\left(\mathrm{CE}_{\mathrm{a}}, \mathrm{pH}, \mathrm{pHc}\right.$, RAS, $\mathrm{Na}^{+}, \mathrm{Ca}^{2+}, \mathrm{Mg}^{2+}, \mathrm{K}^{+}, \mathrm{Cl}^{-}, \mathrm{HCO}_{3}^{-} \mathrm{e} \mathrm{CO}_{3}^{2-}$ ). Também, com os dados de todas as amostras, fêz-se correlações da condutividade elétrica $\left(\mathrm{CE}_{\mathrm{a}}\right)$ com a concentração dos íons e da RAS com a RASaj e RASc. Confeccionou-se um mapa da região estudada locando-se as áreas onde as águas apresentam características semelhantes.

\section{RESULTADOS E DISCUSSÃO}

A Tabela 1 e a Figura 1 mostram que os valores médios da $\mathrm{CE}_{\mathrm{a}}$ variam entre as regiões delimitadas, fato também observado para outras características das águas avaliadas. As áreas (regiões) que apresentaram maior salinidade foram aquelas representadas pelas localidades Apodi (Maisa) e Paulicéia (3,0 $\left.\mathrm{dS} \mathrm{m}^{-1}\right)$, pela localidade Cajazeiras $\left(2,56 \mathrm{dS} \mathrm{m}^{-1}\right)$ e pelas localidades Ibisa (Maisa), União (Maisa) e Pau-Branco, (2,16 $\mathrm{dS} \mathrm{m}^{-1}$ ), sendo que, para irrigação convencional, culturas sensíveis a moderadamente sensíveis à salinidade podem reduzir seu rendimento de forma significativa (Ayers \& Westcot, 1999). Os teores de cloreto nas águas dessas áreas estão acima de $9,0 \mathrm{mmol}_{\mathrm{c}} \mathrm{L}^{-1}$, se enquadrando como águas de restrições severas quanto a toxicidade deste elemento (Ayers \& Westcot, 1999) não sendo recomendadas para irrigar culturas sensíveis ao cloreto.

Confrontando o mapa hidrogeológico da região estudada (SUDENE, 1980) com o mapa da salinidade da água (Figura 1), observa-se que os menores valores de salinidade estão na área da Formação Calcário Jandaíra, enquanto as mais salinas estão na área da Formação Grupo Barreira. Isso pode ser 
RAScor podem ser estimadas com precisão $\left(R^{2}>0,90\right)$ a partir da RAS, sendo a RASaj e RAScor, respectivamente, 2,74 e 1,51 vezes a RAS, constantes essas superiores às verificadas por Medeiros (1992), Martins (1993) e Oliveira \& Maia (1998). Isso pode ser explicado pelo fato das águas de origem calcária serem muito mais ricas em $\mathrm{HCO}_{3}{ }^{-} \mathrm{e} \mathrm{Ca}^{2+}$ que aquelas estudadas pelos autores supra citados.

Tabela 2. Relação entre diferentes características da água de irrigação

\begin{tabular}{ccc}
\hline Relação & Equação & $\mathrm{R}^{2}$ \\
\hline$\left(\mathrm{Ca}^{2+}+\mathrm{Mg}^{2+}\right) \times \mathrm{CE}_{\mathrm{a}}$ & $\mathrm{Y}=4,75 \mathrm{X}+4,56$ & 0,773 \\
$\mathrm{Ca}^{2+} \times \mathrm{CE}_{\mathrm{a}}$ & $\mathrm{Y}=3,29 \mathrm{X}+3,74$ & 0,813 \\
$\mathrm{Na}^{+} \times \mathrm{CE}_{\mathrm{a}}$ & $\mathrm{Y}=2,60 \mathrm{X}-0,20$ & 0,813 \\
$\mathrm{Cl}^{-} \times \mathrm{CE}_{\mathrm{a}}$ & $\mathrm{Y}=8,76 \mathrm{X}-6,07$ & 0,945 \\
RAS x CE & $\mathrm{Y}=0,71 \mathrm{X}+0,44$ & 0,624 \\
RASaj x RAS & $\mathrm{Y}=2,74 \mathrm{X}$ & 0,983 \\
RASc x RAS & $\mathrm{Y}=1,51 \mathrm{X}$ & 0,901 \\
\hline
\end{tabular}

\section{CONCLUSÕES}

1. Existem diferentes regiões homogêneas, cada uma com valores de salinidade e composição da água específicos com salinidade variando de 1,17 a $2,98 \mathrm{dS} \mathrm{m}^{-1}$.

2. Em algumas áreas, a toxidade do cloreto pode afetar, de forma significativa, o rendimento de culturas sensíveis a moderadamente sensíveis.

3. As águas apresentam baixa sodicidade e elevada alcalinidade.

\section{LITERATURA CITADA}

Ayers, R.S.; Westcot, D.W. A qualidade de água na agricultura. 2.ed. Campina Grande: UFPB, 1999, 153p. FAO. Estudos Irrigação e Drenagem, 29 revisado 1

Costa, R.G. Caracterização da qualidade de água de irrigação na microrregião homogênea de Catolé do Rocha (MRH 89). Campina Grande: UFPB, 1982. 89p. Dissertação Mestrado

Cruz, W.B.; Melo, F.A.C.F. de. Zoneamento químico e salinização das águas subterrâneas do Nordeste do Brasil. In: Boletim de Recursos Naturais - SUDENE. v.7, n.1/4, p.7-40, 1969.

EMBRAPA - Empresa Brasileira de Pesquisa Agropecuária. Manual de métodos de análise de solos. Rio de Janeiro, 1997. 212p.

Egreja Filho, F.B., Maia, C.E.; Morais , E.R.C. Método computacional para correção da alcalinidade de águas para fertirrigação. Revista Brasileira de Ciência do Solo, Brasília, v.23, n.2, p.415-423, 1999.
Laraque, A. Estudo e previsão da quantidade de água de açudes do Nordeste semi-árido brasileiro. Recife: SUDENE, 1989. 95p. Série Hidrológica, 26

Leprun, J.C. Primeira avaliação das águas superficiais do Nordeste: Relatório final do Convênio Manejo e Conservação do Solo do Nordeste Brasileiro. Recife: SUDENE, DRN, 1983. p.91-141. Convênio SUDENE/ORSTON

Martins, L.H. Avaliação da qualidade da água nos mananciais superficiais disponíveis para irrigação na zona Oeste Potiguar. Mossoró: ESAM 1993. 65p. Monografia de PósGraduação latu sensu

Medeiros, J.F. de. Qualidade de água de irrigação e evolução da salinidade nas propriedades assistidas pelo GAT nos Estados do RN, PB e CE. Campina Grande: UFPB, 1992. 173p. Dissertação Mestrado

Medeiros, J.F. de; Gheyi, H.R. Manejo do sistema solo-águaplanta em solos afetados por sais. In: Gheyi, H.R; Queiroz, J.E.; Medeiros, J. F. de. Manejo e controle da salinidade na agricultura irrigada. Campina Grande: UFPB, SBEA, 1997. Cap. 8, p.239-284.

Nakayama, F.S.; Bucks, D.A. Trickle irrigation for crop production: Design, operation and management. Amsterdam: Elsevier Science Publishers, 1986. 383p.

Oliveira, O.; Maia, C.E. Qualidade físico-química da água para a irrigação em diferentes aquíferos na área sedimentar do Estado do Rio Grande do Norte. Revista Brasileira de Engenharia Agrícola e Ambiental, Campina Grande, PB, v.2, n.1, p.17-21, 1998.

Palacios, O.; Aceves, E. Instructivo para el muestreo registro de datos e interpretación de la calidad del agua para riego agricola. Chapingo: Colegio de Postgraduados-Escuela Nacional de Agricultura, 1970. 47p.

Richards, L.A. (ed) Diagnosis and improvement of saline and alkali soil. Washington: United States Salinity Laboratory Staff, 1954. 160p. USDA. Handbook, 60

Silva Júnior, L.G.A.; Gheyi, H.R.; Medeiros, J.F. Composição química de águas do cristalino do Nordeste Brasileiro. Revista Brasileira de Engenharia Agrícola e Ambiental, Campina Grande, PB, v.3, n.1, p.11-17, 1999.

SUDENE - Superintendência de Desenvolvimento do Nordeste. Mapa cartográfico. 2.ed. Recife, 1972. Escala 1:100000. Folhas SB-24-X-A-VI, SB-24-X-B-IV, SB-24-X-C-III, SB-24-X-D-I.

SUDENE - Superintendência de Desenvolvimento do Nordeste. Plano de aproveitamento integrado dos recursos hídricos do Nordeste do Brasil - Fase I. Recursos hídricos I: Águas subterrâneas. Recife, 1980. Vol. 4. s.p. 